\title{
GEOMETRY, BINARY DIGITS, TOPOLOGY, BANDGAP IMPLANTS \& ELECTRONIC GENETIC ENGINEERING
}

Rodney Bartlett, Australia - This was not written at any institution

\begin{abstract}
-
The article begins with the physics, cosmology and geometry l've been working on for several years (part of the present article uses paragraphs from that earlier work, so you might find them online - there are links to the earlier work on my ORCID page, https://orcid.org/0000-0003-2240-3743). Since the science of Isaac Newton in the second half of the $17^{\text {th }}$ century, we've regarded gravity as the product of mass. Then early last century, Albert Einstein's theory of General Relativity revolutionized understanding of gravity - and four years after that (in 1919), he published a paper titled "Do gravitational fields play an essential role in the structure of elementary particles?" Considering Einstein's contributions to science is enough to make us wonder if we should instead regard mass as the product of gravity. That's what the first section of this article does, via a simple mathematics which the author developed for his own comprehension of various sciences, and which he calls "vectortensor-scalar geometry". The second section proposes that the entire universe (as well as everything in time) is digital in nature, being composed of electronics' binary digits that enter a shortcut through time (a wormhole) in our far future and build the Mobius strips, figure-8 Klein bottles, and adaptive Wick rotation composing space-time. (This section also provides a quantum explanation of the superflow in supersolids.) The final third section speaks of bandgap implants in the brain, and unites them with the first two parts. Then gravitational fields play an essential - indeed, formative - role in the structure of all types of matter plus its strong and weak nuclear forces. In an extension of Morpho butterflies' ability to selectively add and delete wavelengths of light, this enables the implants to selectively add and delete "wavelengths of matter" e.g. in future, electronic genetic engineering. Union with the digital universe means surgery will oneday be unnecessary to obtain an implant - it'll simply be a downloaded program.
\end{abstract}

\section{Keywords -}

Gravity; Mass; Geometry; Binary digits; Topology; Wick rotation; Bandgap implants; Future genetic engineering; Regenerative medicine; Bioartificial organs; Supersolids 


\section{Article -}

\section{VECTOR-TENSOR-SCALAR GEOMETRY}

Two adjoining sides of a parallelogram (see Fig. 1) represent the vectors of the photon's spin 1 and the graviton's spin 2 . The resultant diagonal represents the interaction of the sides/vectors $(1 \div 2=$ the spin $1 / 2$ of every matter particle). Tensor calculus changes the coordinates of the sides and diagonal into the coordinates of a position on a line (a single point on the diagonal). This scalar point is associated with particles of spin 0 . If the mass produced during the $1 \div 2$ interaction happens to be $125 \mathrm{GeV} / \mathrm{c}^{2}$, its union with spin 0 produces the Higgs boson. $125 \mathrm{GeV} / \mathrm{c}^{2}$ united with spin 0 means the central scalar point of the Higgs boson is related to the vector of the graviton's spin 2, and the Higgs field is therefore united with the supposedly unrelated gravitational field (together with the latter's constant interaction with the electromagnetic field).

One of parallelogram's horizontal sides -

represents the electromagnetic component

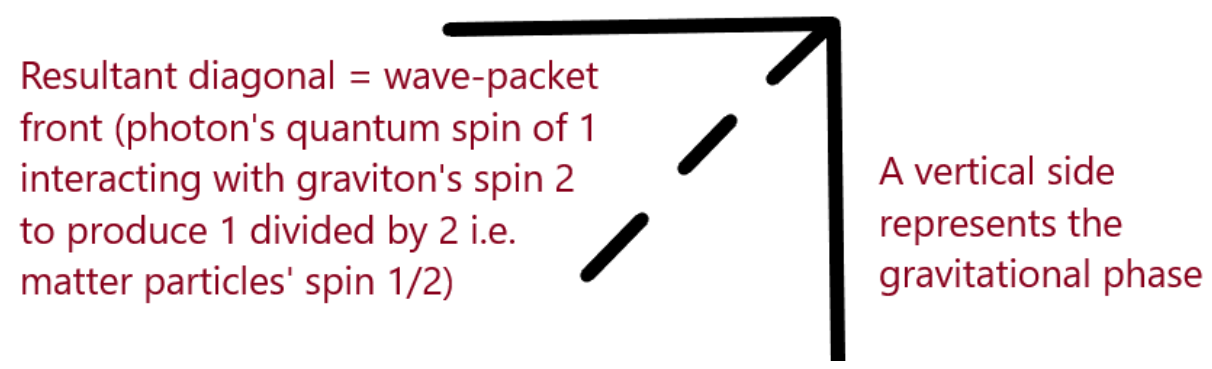

FIGURE 1: WAVE-PACKET FRONT AS QUANTUM SPIN

It must be remembered that referring to space alone is incomplete. Living in space-time, it's necessary to add some sentences about the time factor. The photon must interact with the graviton to produce the mass of the mass of the weak nuclear force's $W$ and $Z$ bosons. To produce their quantum spin, the photon's spin 1 needs to react with the graviton's spin 2. That is, the photon's turning through one complete revolution needs to be combined with the graviton's being turned through two half-revolutions.^ Incorporating the time factor as a reversal of time in the middle of the interaction: a gravitonic half revolution is subtracted from the photonic full revolution then the graviton's time-reversal adds a half revolution $(1-1 / 2+1 / 2=$ the spin 1 of $W$ and $Z$ bosons). The strong nuclear force's gluon's quantum spin of 1 could arise in the same way as the spin 1 of weak-force bosons. 
$\wedge$ Professor Hawking writes,

"What the spin of a particle really tells us is what the particle looks like from different directions." (1)

Spin 1 is like an arrow-tip pointing, say, up. A photon has to be turned round a full revolution of 360 degrees to look the same.

Spin 2 is like an arrow with 2 tips - 1 pointing up, 1 down. A graviton has to be turned half a revolution (180 degrees) to look the same.

Spin 0 is like a ball of arrows having no spaces. A Higgs boson looks like a dot: the same from every direction.

Spin $1 / 2$ is logically like a Mobius strip, though Hawking doesn't specifically say so. This is because a particle of matter has to be turned through two complete revolutions to look the same, and you must travel around a Mobius strip twice to reach the starting point.

Referring to the diagonal in Fig. 1: since mass (and the nuclear forces associated with matter) are a product of gravitation, the gravitational waves do not simply penetrate matter but - like a biological enzyme - must pause to react with it. The matter absorbs and re-radiates the gravitational waves so rapidly that they appear to merely pass through the matter unimpeded

\section{BITS AND TOPOLOGY}

These scientists support the idea of the universe being composed of information/mathematics:

a) In 1990, John Wheeler (1911-2008) suggested that information is fundamental to the physics of the universe. According to this "it from bit" doctrine, all things physical are information-theoretic in origin. (2)

b) Erik Verlinde says gravity is not a fundamental force of nature, but an emergent phenomenon. In the same way that temperature arises from the movement of microscopic particles, gravity emerges from the changes of fundamental bits of information, stored in the very structure of spacetime. (3) 
c) Cosmologist Max Tegmark hypothesizes that mathematical formulas create reality. (4)

d) "Pioneered (in the late 1980's) by Rafael Sorkin, a physicist at the Perimeter Institute in Waterloo, Canada, the theory (causal sets) postulates that the building blocks of space-time are simple mathematical points that are connected by links, with each link pointing from past to future." (5)

If present-day physicists like Erik Verlinde and Max Tegmark are correct in thinking the universe has a mathematical foundation, that foundation could be the electronic BITS (BInary digiTS) of 1 and 0 , which comprise what is known as base- 2 mathematics. The exactness of binary calculation would then be the information which biochemist Christian Anfinsen determined was residing in the sequence of amino acids, and is necessary for protein folding. (6) The exactness could also change present understanding of quantum mechanics by deleting probability in subatomic reactions ensuring that if enzymes use quantum tunneling (where a particle passes through a barrier that it classically cannot penetrate) to accelerate biochemical reactions, (7) those enzymes are guaranteed to function properly within the body and will not merely have a probability of being successful.

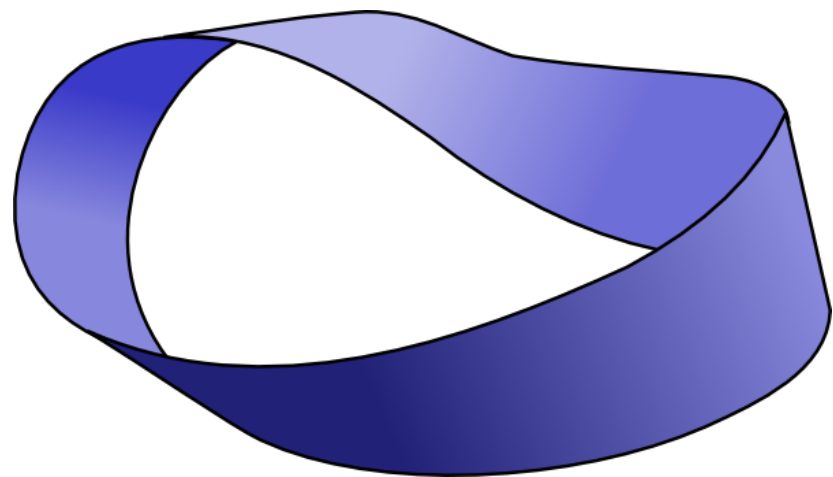

Figure 2 - the Mobius Strip, which is two-dimensional and only has one surface (source: http://www.clker.com/cliparts/3/7/ a/9/1220546534781713951lummie Mobius Strip.svg.hi.png)

How could this information in the form of binary digits produce specific shapes that fit together like a lock and key? First, the 1's and 0's form the shape of a Mobius strip, which is merely two-dimensional (2-D). To use words from a recent paper

In a holographic universe, all of the information in the universe is contained in 2D packages trillions of times smaller than an atom. (8) 
("Holographic" would have the accepted cosmological meaning of the entire universe being seen as two-dimensional information - from Mobius strips, according to this article - projected into the three dimensions we're familiar with.) Then two strips must be joined to make a Klein bottle (9) which has the 3 dimensions of length, width, depth and the 4th dimension of movement in time. The type of Klein bottle formed would appear to be the figure-8 Klein. A diagram of many figure-8 Klein bottles would show that their positive curvature (on the spherical parts) fits together with their negative curvature (on saddle-shaped parts) to cancel and produce, on a cosmic scale, the flat curvature of space-time (10).

When you have trillions of Mobius and figure-8 Klein elements assembled, you can follow the theory of the mass-giving Higgs field being the result of various couplings. (11) This theory has lost popularity since the Higgs boson was discovered. But an implication of a 1919 paper by Einstein called "Do gravitational fields play an essential role in the structure of elementary particles?" (12) is that the coupling is between gravitons (the hypothetical quantum units of gravity) and photons (the quantum units of electromagnetism such as visible light, radio waves and X-rays). That could mean coupling is also between the Mobius strips and figure-8 Klein bottles composing the particles. It only needs to be assumed that these topological shapes exist on a level between photons/gravitons and 1's/0's, being built up into the particles and being composed of the binary digits). With trillions of Mobius and figure-8 Klein elements assembled, these (now respectively called photons and gravitons) must interact to give matter what we call the emergent property of mass - similar to hydrogen and oxygen combining to give water what we call wetness. This proposed link between the Mobius strip and the Mobius doublet (figure-8 Klein bottle) would also be a link between the photon and graviton - suggesting the unification of electromagnetism with gravitation, which Einstein sought in his Unified Field Theory (he died before completing the theory). It also confirms Erik Verlinde's idea that gravity is an emergent property (emerging from mathematics). 


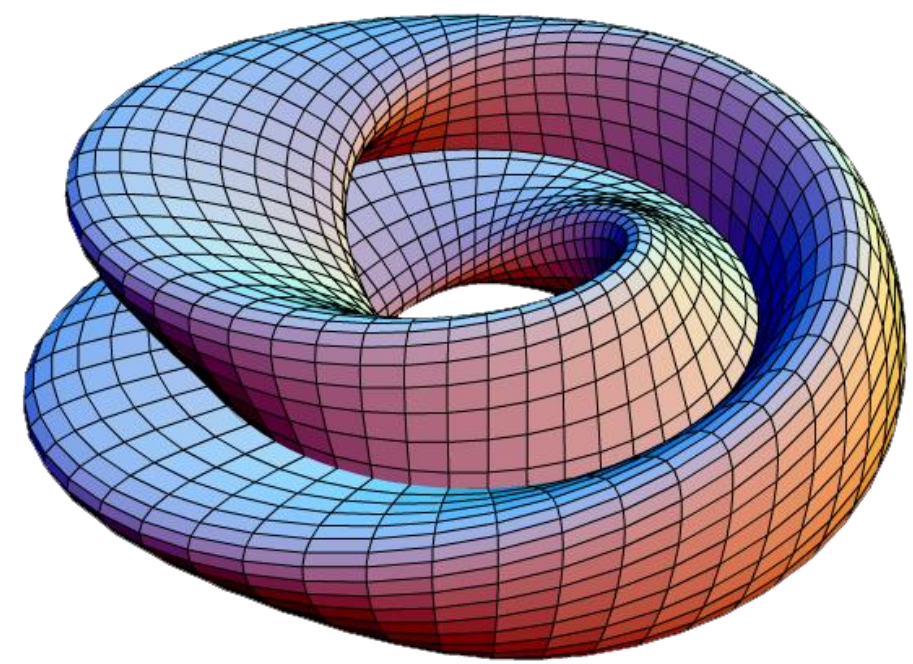

Figure 3 - MOBIUS DOUBLET (FIGURE-8 KLEIN BOTTLE) (source:

https://upload.wikimedia.org/wikipedia/commons/7/73/KleinBottleFigure8-01.png) Note that, when considering many bottles, the reddish positive curvature fits together with the bluish negative curvature to produce the flatness implying space-time's infinity and, since space and time are always unified, its eternity. (In flat space-time, light beams travel in straight lines and can go infinite distance without ever meeting.)

Following Albert Einstein's example of turning Max Planck's quanta (which, for years, Planck and all other scientists considered purely mathematical) into explanation of the physical photoelectric effect, the Wick rotation used to describe imaginary time may be transformed from mathematical "trickery" to physical meaning, and provide a modern way to unite space and time into one space-time. 


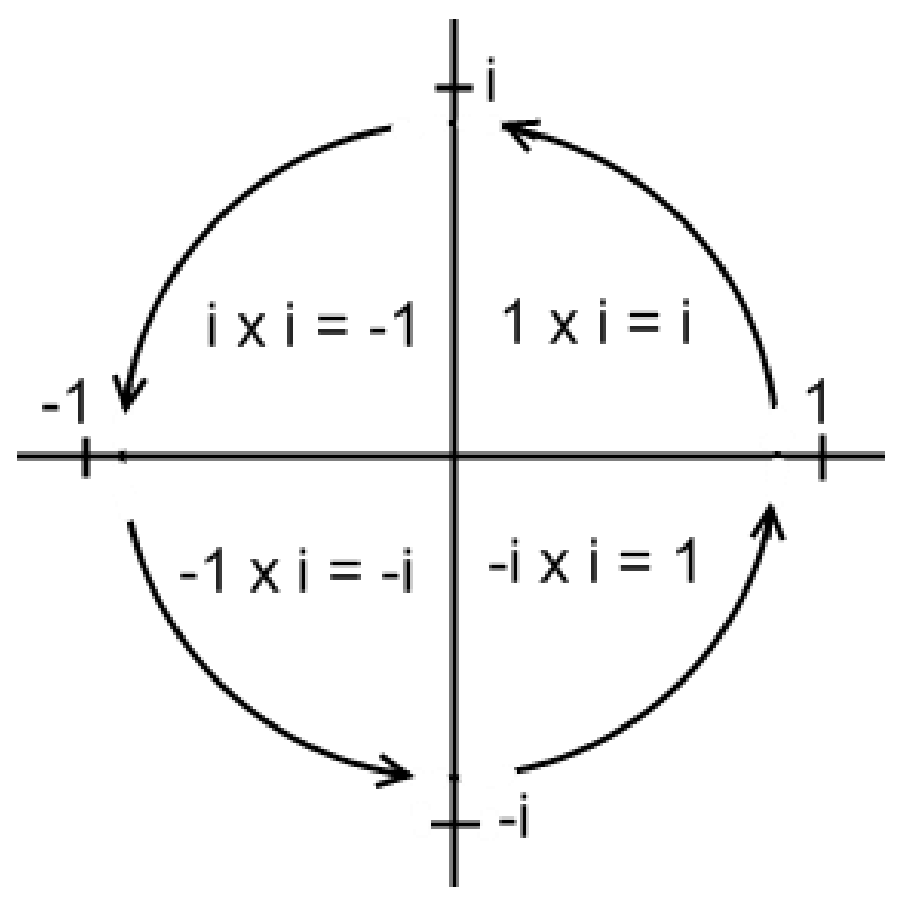

Figure 4-WICK ROTATION: "The complex plane reveals i's special relationship with cycles via the circle of $i$, also known as Wick rotation. Whenever a point on the complex plane is multiplied by $\mathrm{i}$, it moves a quarter rotation around the origin or center of the plane." [13]

This submission claims Mobius strips compose energy particles as well as matter particles (see "Spin $1 / 2$ is like a Mobius strip", p. 3) and the Wick rotation inherent in the strips is adaptive. There are vastly increased numbers of gravitational energy's gravitons near black holes, and there is increased effect on electromagnetic energy's photons when speed-of-light travel includes vast numbers of photons in the traveller's sphere of influence. These enlarged quantities boost the probability of Wick rotations cancelling to produce quantum entanglement. "Advanced" waves travel back in time (to the left of Fig. 4's origin or centre). "Retarded" waves go forwards in time (to the right of Fig. 4's centre). Imagine the wave below as a retarded wave originating at the red dot on the far left. As it rotates around the origin in Fig. 4's anticlockwise direction to occupy coordinates proceeding to the left of the origin, its crests become troughs and troughs become crests (the other red dots mark the straight lines of the axes). In this way, the crests and troughs cancel each other and cancellation produces quantum mechanics' entanglement. In other words, a wave interferes with itself (and a particle - whether boson or fermion - formed from gravitational and electromagnetic waves interacts with itself). This interference of particles is demonstrated by the Mach-Zehnder interferometer since it splits light beams, causes travel in different directions, and recombines the photons. Interference of particles with themselves is also demonstrated by supersolids (solids that have superfluid - frictionless - properties and can flow through themselves). Their "superflow" (frictionless flow) may not be due to actual fluid 
moving along imperfections but to a special quantum state (where particles interfere with themselves) being formed at a low temperature very close to absolute zero.

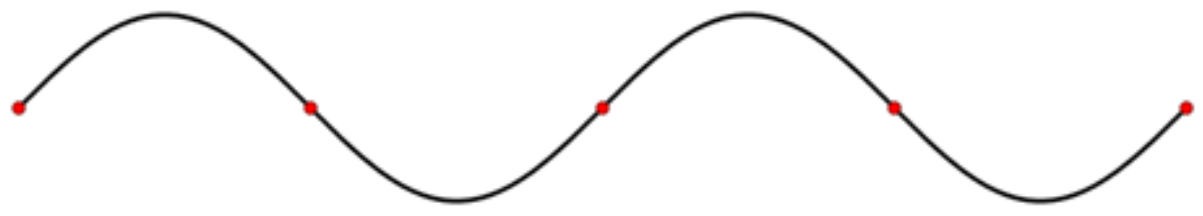

Figure 5 - Wave (Public domain image from https://en.wikipedia.org/wiki/Wave\#/media/File:Standing wave.gif)

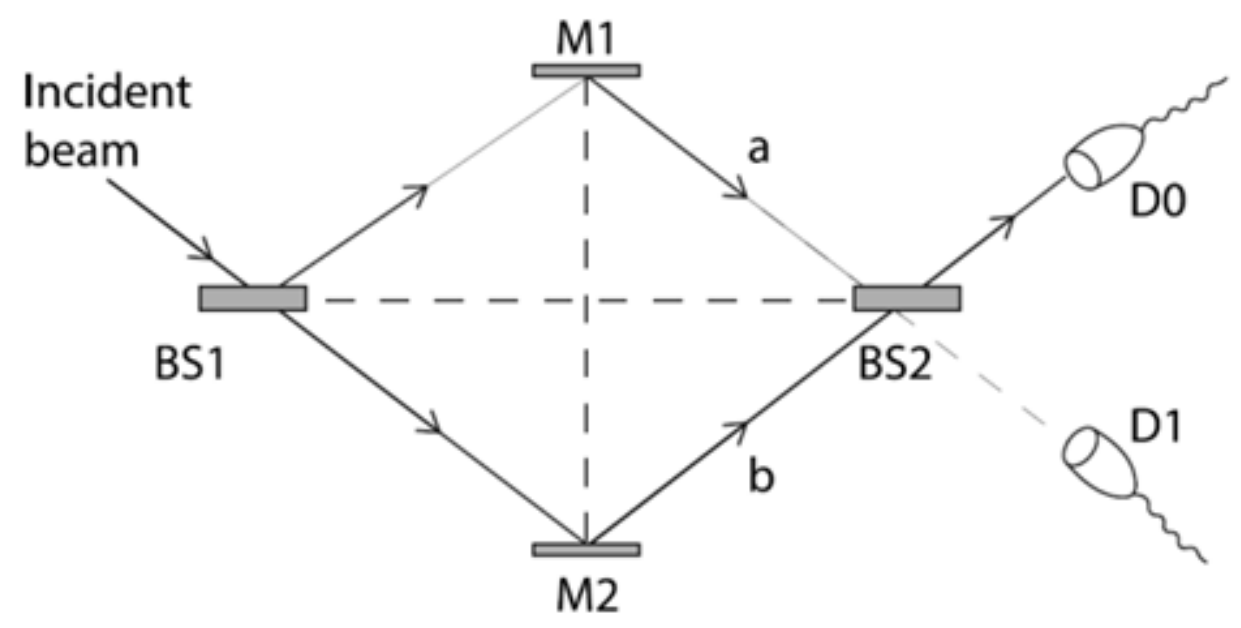

Figure 6 - Mach-Zehnder interferometer

We have two beam splitters (BS1 and BS2) and two perfect mirrors (M1 and M2). An incident beam coming from the left is split at BS1 and recombines at BS2, which sends two outgoing beams to the photon detectors D0 and D1. The interferometer can be set up to produce a precise interference effect which ensures all the light goes into D0, as shown above. Alternatively, the setup may be altered to ensure all the light goes into D1.

(Source of figure and figure's text - "Reading Feynman" by Jean Louis Van Belle: https://readingfeynman.org/tag/interference-of-a-photon-with-itself/. His reference for the illustration is MIT edX Course 8.04.1x (Quantum Physics), Lecture Notes, Chapter 1. Section 4 (Quantum Superpositions). 
As well, an effect of gravitational and electromagnetic waves following Wick rotation is to cause cancelling crests and troughs to produce Special Relativity's time dilation (slowing - with eventual stoppage at the speed of light) near the intense gravity of black holes, and near light speed. They also move the idea of waves travelling back in time into agreement with verified Special Relativity and quantum mechanics.

The Mobius Doublet (Figure-8 Klein Bottle) has space-time's four dimensions when adaptive Wick rotation is added to Mobius strips, manifesting as Special Relativity's non-absolute time. About the adaptive nature of Wick rotation: "Physicists now believe that entanglement between particles exists everywhere, all the time, and have recently found shocking evidence that it affects the wider, 'macroscopic' world that we inhabit" (14). "Caslav Brukner, working with Vlatko Vedral and two other Imperial College researchers, has uncovered a radical twist. They have shown that moments of time can become entangled too" (15). When the figure-8 Klein bottles (which contain Wick rotation in their constituent Mobius strips) encounter other figure-8's, there's a probability that their rotations will cancel because of entanglement (one rotation may be clockwise with the other being anticlockwise), slowing and even stopping time. As the number of figure-8's accumulates, the chance of time's cessation increases. Think of a black hole as a focus for gravitational waves. The gravitons composing the waves are themselves composed of figure-8's. As these Klein bottles accumulate, time slows (the time dilation is lessened with bodies of reduced mass ie bodies such as stars and planets which are the focus of fewer, less powerful waves). In agreement with Relativity, intense gravity slows time. Speeds approaching $c$ (the velocity of light in a vacuum) do the same. Great speeds affect great numbers of Wick-rotation-containing Mobius strips and figure-8 Klein bottles (composers of, respectively, the universe's entanglement of photons and gravitons) resulting in increased chance of time slowing due to cancelling rotations. In conclusion; if gravitons and photons are entangled, so are the figure- 8 Klein bottles and Mobius strips and Wick rotations that compose the particles.

\section{BANDGAP IMPLANTS}

"In solid-state physics, a band gap, also called an energy gap or bandgap, is an energy range in a solid where no electric current can exist. In graphs of the electronic band structure of solids, the band gap generally refers to the energy difference (in electron volts) between the top of the valence band and the bottom of the conduction band in insulators and semiconductors. It is the energy required to promote a valence electron 
bound to an atom to become a conduction electron, which is free to move within the crystal lattice and serve as a charge carrier to conduct electric current. It is closely related to the $\mathrm{HOMO} / \mathrm{LUMO}$ gap^ in chemistry. If the valence band is completely full and the conduction band is completely empty, then electrons cannot move in the solid; however, if some electrons transfer from the valence to the conduction band, then current can flow. Therefore, the band gap is a major factor determining the electrical conductivity of a solid. Substances with large band gaps are generally insulators, those with smaller band gaps are semiconductors, while conductors either have very small band gaps or none, because the valence and conduction bands overlap." (16 Wikipedia article used for background information only)

^ "highest occupied molecular orbital and lowest unoccupied molecular orbital, respectively. "molecular orbital" is a mathematical function describing the wave-like behavior of an electron in a molecule. The energy difference between the HOMO and LUMO is termed the HOMO-LUMO gap."

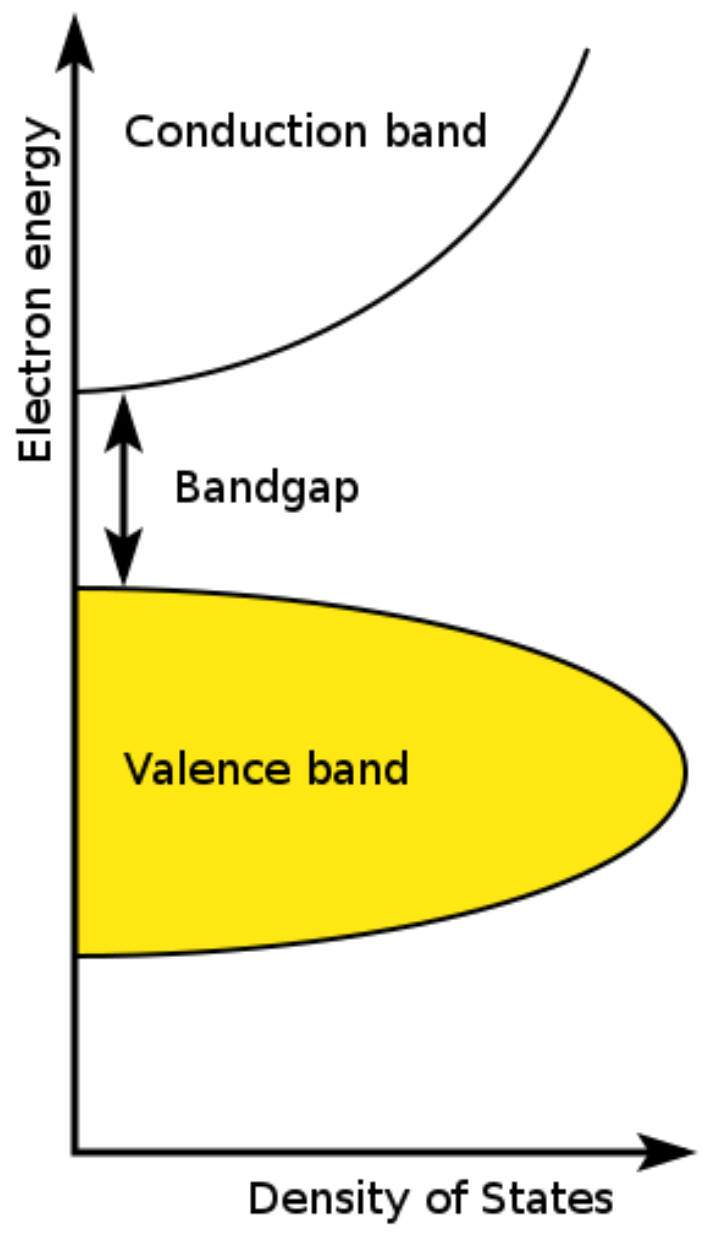


Figure 7 - A sketch of the bandgap between valence band and conduction band in insulators and semiconductors. (Public Domain image)

"(Morpho) butterflies create color by selectively adding and deleting certain wavelengths of light. Physicists have only recently devised comparable materials, called photonic band-gap crystals; and are now exploring their use in phone switches, solar cells and antennas. No surprise, then, that some engineers are looking to the living world for the next generation of optic inspirations." (quote from 17)

I believe advances in engineering and biology will enable humans, like the morpho butterfly, to selectively add and delete wavelengths of light. But the word "light" need not only refer to visible wavelengths. It can be extended and refer to any wavelength of the electromagnetic spectrum. Science accepts that radio, infrared, ultraviolet waves, $X$ rays and gamma radiation are all forms of light. Suppose matter acquires all its properties (including mass) by the interaction of electromagnetic and gravitational waves - the day will come when we can add or delete wavelengths of matter, anywhere and anytime we choose!

I anticipate people will oneday have band-gap structures in their brains that are no bigger than a computer chip (these won't require surgical implantation, but simply downloading, because of the pre-existing digital nature of all parts of the universe). Photonic band-gap crystals would, of course, only deal with light in its photonic forms (energy forms such as visible light or radio waves). The band-gap structures I have in mind would need to deal with forms of matter like genes. They could add or delete anything and everything we choose by emulating computers' copy/paste function to add things; as well as their delete function, to remove things (now that's what I call genetic engineering!)

\section{REFERENCES}

(1) Stephen Hawking, 1988, 'A Brief History of Time', pp.66-67 - Bantam Press

(2) Wheeler, John A (1990) "Information, physics, quantum: The search for links". In Zurek, Wojciech Hubert. Complexity, Entropy, and the Physics of Information. Redwood City, California: Addison-Wesley. 
(3) E P Verlinde (7 Nov 2016) "Emergent Gravity and the Dark Universe". arxiv.org/abs/1611.02269

(4) Max Tegmark (January 2014) "Our Mathematical Universe". Random House/Knopf.

(5) Zeeya Merali (August 2013) "Theoretical physics: The origins of space and time". ("Nature" 500, 516-519-28).

(6) Anfinsen, Christian B, Edgar Haber, Michael Sela, and F H White Jr. (15 September 1961) "The Kinetics of Formation of Native Ribonuclease During Oxidation of the Reduced Polypeptide Chain." Proceedings of the National Academy of Sciences of the United States of America 47, 9: 1309-1314.

(7) Jim Al-Khalili and Johnjoe McFadden (November 6th 2014) "Life on the Edge: The Coming of Age of Quantum Biology". First published by Bantam Press.

(8) Niayesh Afshordi, Claudio Corianò, Luigi Delle Rose, Elizabeth Gould, and Kostas Skenderis (27 January 2017) "From Planck Data to Planck Era: Observational Tests of Holographic Cosmology". Phys. Rev. Lett. 118, 041301. (https://journals.aps.org/prl/ abstract/10.1103/PhysRevLett.118.041301)

(9) Polthier, Konrad (September 1, 2003) "Imaging maths - Inside the Klein bottle". http://plus. maths.org/content/os/issue26/features/mathart/index

(10) WMAP science team (12/20/2012) "Wilkinson Microwave Anisotropy Probe". https://map.gsfc.nasa.gov

(11) M. Tanabashi, M. Harada, and K. Yamawaki (2006) "The Origin of Mass and Strong Coupling Gauge Theories". "The Origin of Mass and Strong Coupling Gauge Theories". International Workshop on Strongly Coupled Gauge Theories.Nagoya. pp. 227-241.

(12) Albert Einstein (1919) "Do gravitational fields play an essential role in the structure of elementary particles?" ("Spielen Gravitationsfelder im Aufbau der materiellen Elementarteilchen eine wesentliche Rolle?") Sitzungsberichte der Preussischen Akademie der Wissenschaften, [Math. Phys.], 349-356. Berlin.

(13) Figure and quote from "The Meaning of Imaginary Time: Creativity's Dialog with Timelessness" - Posted on July 15, 2015 by Kerri Welch (public domain figure supplied by WordPress) https://textureoftime.wordpress.com/2015/07/15/the-meaning-ofimaginary-time/

(14) "The Weirdest Link": New Scientist, vol. 181, issue 2440 - 27 March 2004, page 32 - online at http://www.biophysica.com/QUANTUM.HTM 
(15) "Quantum Entanglement in Time" by Caslav Brukner, Samuel Taylor, Sancho Cheung, Vlatko Vedral (Submitted on 18 Feb 2004) - http://www.arxiv.org/abs/quantph/0402127

(16) "Band gap", https://en. wikipedia.org/w/index.php?title=Band gap\&oldid=899958022 (last visited June 14, 2019)

(17) "Illuminated Life - Meet the true masters of optics: Animals that know a lot more about slicing, dicing, and twisting beams of light than we do" by George M. Whitesides, Felice Frankel - Discover Magazine, August 2005 issue 